\title{
Computer Simulation, Experiment, and Novelty
}

\author{
Julie Jebeile
}

Institut supérieur de philosophie, Université catholique de Louvain

CONTACT Julie Jebeile / julie.jebeile@uclouvain.be / Institut supérieur de philosophie, Université catholique de Louvain, Collège Mercier, place du Cardinal Mercier, 14, boîte L3.06.01, 1348 Louvain-la-Neuve, Belgium

\begin{abstract}
It is often said that computer simulations generate new knowledge about the empirical world in the same way experiments do. My aim is to make sense of such a claim. I first show that the similarities between computer simulations and experiments do not allow them to generate new knowledge but invite the simulationist to interact with simulations in an experimental manner. I contend that, nevertheless, computer simulations and experiments yield new knowledge under the same epistemic circumstances, independently of any features they may share.
\end{abstract}

\section{Introduction}

Computer simulations have been ubiquitous in science since the rapid growth of computers. They are used to gain knowledge about the target phenomena they are supposed to instantiate. From the 1990s onwards, they then received much attention from philosophers and sociologists of science. A central epistemological question at the time was where to locate computer simulations between theoretical work and experimentation on the 'methodological map' (Galison 1996, 120). Thus one reads: 
[C]omputer simulation provides (though not exclusively) a qualitatively new and different methodology for the physical sciences, and ... this methodology lies somewhere intermediate between traditional theoretical physical science and its empirical methods of experimentation and observation. (Rohrlich 1991, 507)

[C]omputational methods of numerical experimentation constitute a distinctively new kind of scientific method, intermediate in kind between empirical experimentation and analytic theory. (Humphreys 1994, 103)

The ambiguous position of simulation with regard to 'theory' and 'experiment' is widely acknowledged in the scientific literature .... Simulation can be aligned with whichever methodological category suits the local circumstances. (Dowling 1999, 263)

Mathematical models and their cousins, computer simulations, occupy an uneasy space between theory and experiment, between abstract and concrete, and often between the pressures of pure science and the needs of pragmatic action. (Sismondo 1999, 247)

More recently, some philosophers have drawn an analogy between simulations and experiments (e.g. Norton and Suppe 2001; Guala 2002, 2005; Morgan 2002, 2003, 2005; Keller 2003; Winsberg 2003). If such an analogy is justified, then it follows that, based on their similarities, some epistemic properties of experiments can be extended to simulations. This analogy would nicely account for the fact that computer simulations can replace experiments when experiments are too costly, uncertain in their outcomes, too time-consuming, politically unacceptable or ethically undesirable (Humphreys 2004, 107). In these cases, simulations are often called 'numerical experiments' (or 'experimental simulations', 'simulated experiments', 'in silico experiments').

A significant part of the discussions focuses on the knowledge-making role of simulations and experiments. Here it is argued that, based on their similarities, simulations generate knowledge in the same way that experiments do. Thus one finds: 
Simulations can produce new knowledge just as experiments do. (Guala 2002, 1)

Some computer simulations undoubtedly share some epistemic functions with field experiments: they are run to provide new data about systems that are difficult or impossible to investigate with ordinary instruments. (Barberousse, Franceschelli, and Imbert 2009, 557)

[S]imulations involve complex inferences as they move from theory to data, and they certainly generate new knowledge. (Winsberg 2009, 578-579)

Here two claims are generally implicitly intertwined: first, like experiments, simulations generate knowledge that is empirical in nature; second, like experiments, simulations generate new knowledge.

The first claim faces what is sometimes called the 'materiality problem'. Experiments involve, within the matter of the experimental system, the physical processes that produce the target phenomenon being studied; while simulations involve, within the machine, other physical processes that produce the calculations but do not generate the phenomenon itself (Guala 2002). For some authors (e.g. Morgan 2002, 2003; Giere 2009), this makes simulations epistemologically inferior to experiments. Substantial objections, based on distinct arguments, have been nevertheless made (e.g. Norton and Suppe 2001; Barberousse, Franceschelli, and Imbert 2009; Parker 2009; Lusk 2016).

The second claim has received a less systematic philosophical treatment so far (except in Beisbart and Norton 2012; Barberousse and Vorms 2013; Morrison 2015, chap. 7; Lusk 2016). What shall be called here the 'novelty claim' states that computer simulations generate new knowledge in the same way experiments do. This faces the 'non-entailment view' (Lusk 2016, 151) according to which simulations cannot go beyond the code; they just make explicit the consequences of what is contained in the computer program.

In this article, I focus on the novelty claim independently of the question whether, like experiments, computer simulations provide knowledge that is empirical in 
nature. My objective is to make sense of the novelty claim and thereby to get a grasp on the widely shared intuition behind it.

An attempt at making sense of the novelty claim is the analogy between simulations and experiments: one might be tempted to argue that the features they have in common allow them to generate new knowledge. I therefore examine the similarities between simulations and experiments, which are often highlighted in the philosophical literature, and I investigate whether they bestow knowledge-making capability upon them (sections 3 ). I endeavour to explicate the intuition behind the novelty claim (section 4). I then analyse the concept of novelty and, from this, frame an argument that simulations and experiments generally provide new knowledge under the same epistemic circumstances as experiments (section 5).

\section{Some Definitions}

First of all, I need to define the basic terms I use in the article. Scientific models received a great deal of attention in the 1990s, when some philosophers abandoned any foundationalist project such as initiated by the logical positivists and started to focus on scientific practices. An examination of practices indeed leads to value models more than theories. For instance, predicting and explaining do not strictly amount to deducing from a theory the logical consequences following the hypothetical-deductive scheme; these two epistemic activities require in practice to build a model. A model is therefore an application of the theoretical principle at hand to the specific situation being studied that the theory cannot describe alone. It contains not only theoretical principles but also extrinsic components such as abstractions, idealizations, fictional components and ad hoc assumptions. 'Scientific model' is a polysemous term. There have been different accounts of models, i.e., the linguistic view (e.g. Max Black, Mary B. Hesse), the semantic view (e.g. Patrick Suppes, Bas van Fraassen, Ronald N. Giere) and a more recent practices-based account on which this article builds (e.g. Nancy Cartwright, Mary S. Morgan, Margaret Morrison, Michael Redhead).

Simulation models, also called computational models, contain theoretical principles, simplifying assumptions but also mathematical approximations due to the numerical scheme that is required for running calculations on a computer. The program, 
written in a computer language, contains the algorithm which describes how to process calculations from the simulation model. 'Computer simulation' designates these calculations but sometimes refers in the literature to the simulated phenomenon on the computer screen.

There are different types of simulation models depending on the underlying numerical methods, e.g. discretisation-based numerical methods, cellular automata, and agent-based models. Since most of the simulation models used in empirical science (e.g. physics, chemistry, biology) represent empirical systems with the means of partial differential equations, I focus in this article on discretisation-based simulations.

Now that I have made clear what I mean by models and simulations, I will now explore the features that simulations and experiments share.

\section{Similarities between Simulations and Experiments}

If the analogy between simulations and experiments is justified, it should follow that, based on their similarities, simulations generate new knowledge in the same way experiments do. In this section, I will take this analogy seriously. I first review five similarities between simulations and experiments that are often highlighted by philosophers. And I then question whether these similarities allow simulations and experiments to generate new knowledge (along the lines of Jebeile 2016, 61-67). Note that the list of similarities may not be exhaustive and that I do not mean that, when providing new knowledge, simulations and experiments are supposed to exhibit all these features at the same time.

\subsection{Exploration}

First, it is often said that simulation and experiment both allow for exploration. Scientific exploration - in simulations or experiments - is about changing some input conditions and control parameters, and examining how the system under study reacts to this change. This change often aims at answering a specific question the scientist raises. Experiment consists in exploring the phenomena by providing observations and measures, while simulation consists in mathematically exploring the theoretical 
implications of the simulation model. In this sense, Dowling (1999), Morgan (2002), and Keller (2003) call simulations 'experiments on the theory' (or on the model); simulations are also sometimes called 'virtual laboratories'.

For example, in the simulation of a flow past a solid cylinder, the simulationist may be interested in knowing under which physical conditions vortices are induced by the interaction between the fluid and the cylinder. In order to do this, she can change the value of the fluid inflow velocity until vortices appear. More generally, in the case of a simulation, the simulationist enters into the program the initial conditions and the parameter values that are relevant to the question, and then collects the simulation outputs. If these outputs fail to provide a satisfying and complete answer to the question, the simulationist can run another simulation with other conditions and parameter values. Each time she gets new outputs that may in turn provide new knowledge about the system's behaviour.

This interaction of the 'question/answer' sort is the same between the experimenter and the experimental system. The experimenter changes some parameters and observes the reaction of the system to this change. For example, in the experiment of a flow past a solid cylinder, the experimenter can change the value of the input parameters, such as the input mean fluid velocity, in the water tunnel. The interaction of the 'question/answer' sort is possible because the computer program, like an experimental device, creates a controlled environment. That is why Margaret Morrison claims that the simulation system - which includes the computer, the program and the simulation model - 'functions like a piece of laboratory equipment, used to measure and manipulate physical phenomena' (Morrison 2009, 45). In particular, 'Considered as an apparatus it allows us to create the kind of controlled environment where one can vary initial conditions, values of parameters etc.' (Morrison 2009, 44-45).

\subsection{Intervention}

Exploration implies that a scientist intervenes to some extent on the computer program or the experimental setup. Thus simulation and experiment share a second feature in that scientists intervene on them. This similarity is emphasized by Morgan (2002) and Parker (2009). 
Like the philosophers of experiment Hacking (1983), Tiles (1993), Radder (1996), and Woodward (2003), Morgan and Parker insist on the fact that experiment always implies intervention. In particular, Parker specifies, 'An experiment can be characterized as an investigative activity that involves intervening on a system in order to see how properties of interest of the system change, if at all, in light of that intervention'. She adds, 'An intervention is, roughly, an action intended to put a system into a particular state, and that does put the system into a particular state, though perhaps not the one intended' (Parker 2009, 487).

In concrete terms, an intervention on an experimental system is an action that consists, for example, in pressing buttons on the machine, opening valves, heating the system or removing one of its components. In the case of simulations, an intervention on the computer program is an action that consists, for example, in using the mouse to click on screen or in using the keyboard to enter input data.

Interventions therefore entail interactions between the simulationist and the computer program or the experimental system. Therefore it might be better to refer to 'computer simulation studies' rather than to simulations. As Parker highlights, 'computer simulations consist of sequences of computer states-not activities undertaken by inquiring agents' (Parker 2009, 488), while experiments involve agents in intervening on the experimental system. Therefore, as she suggests, it is more convenient to compare experiment with computer simulation study, which is

the broader activity that includes setting the state of the digital computer from which a simulation will evolve, triggering [its] evolution by starting the computer program that generates the simulation, and then collecting information regarding how various properties of the computing system, such as the values stored in various locations in its memory or the colors displayed on its monitor, evolve in light of the earlier intervention (i.e. the intervention that involves setting the initial state of the computing system and triggering its subsequent evolution). (Parker 2009, 488)

That said, in this article, I still use the term simulation while including such activities.

\subsection{Visualization}


Two other similarities between simulation and experiment that are identified in the literature can be illustrated by an example of an astrophysics simulation. This simulation helped astrophysicists to explain the discovery of VirgoHI21 made some years ago. VirgoHI21 was first identified as a 'dark' galaxy because it contains only gas and has no star. Such a discovery contradicts cosmological theory since VirgoHI21 is as massive as the Milky Way while theoretically there is no galaxy without a star as massive as VirgoHI21. Thus, in order to explain this prima facie contradiction, the formation of VirgoHI21 has been reproduced by simulation (Duc, Bournaud, and Brinks 2007). For that purpose, astrophysicists designed a possible scenario of the formation based on empirical observations. In their observations, they noticed that VirgoHI21 is not secluded but located near a spiral galaxy called NGC 4254 (or Messier 99). More precisely, VirgoHI21 forms a long gas filament behind NGC 4254. For the astrophysicists, this provided evidence that VirgoHI21 could be debris from past interaction between two galaxies. Indeed long gas filaments - called 'tidal tails'-are often observed after two galaxies collide; they are constituted of the debris, i.e., pieces, of disk components extracted by tidal forces. Astrophysicists therefore tried to find the right conditions of the collision before VirgoHI21's formation. Figure 1 shows the final result of this simulation.
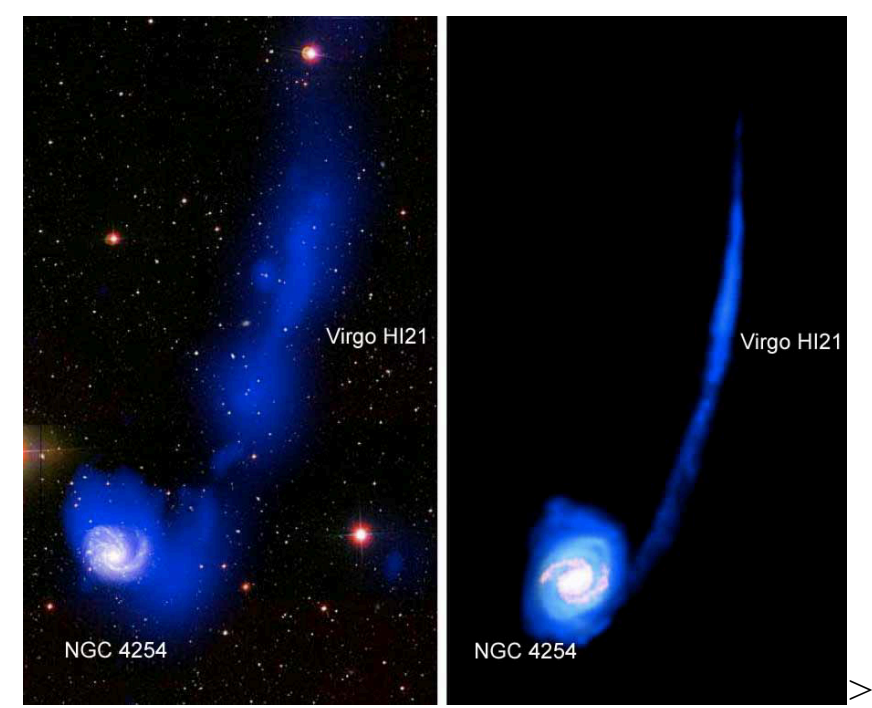

Figure 1. Left: VirgoHI21 and NGC 4254 observed by telescope. Right: the simulation picture. Credits CEA/Sap. 
The simulation accurately recreates the gas distribution and concentration in VirgoHI21. Thus it shows that, under certain conditions, when a galaxy at high speed (around $1000 \mathrm{~km} / \mathrm{s}$ ) comes close to another galaxy, gaseous matter is ejected at large distances and re-condensed in the intergalactic area, taking the form of isolated rotating clouds. The simulation pictures suggest that VirgoHI21 formed 750 millions of years ago after a collision between two spiral galaxies. VirgoHI21 is therefore not a galaxy but only collision debris (Duc, Bournaud, and Brinks 2007). Because of its high speed, the second galaxy — which is most likely Messier 98 - has already gone very far away.

Figure 2 shows different sequences of the simulation film. These sequences have not been captured at identical intervals. Nevertheless, they represent the main evolution steps in the simulation film (which lasts a few seconds). It can be seen, from sequence 1 to 6 , a high-speed galaxy coming close to NGC 4254 . Then, from sequence 7 to 16 , we observe the formation of a tidal tail; note that the second galaxy is out of the picture from sequence 11 but keeps moving far away. This tail, which is now called VirgoHI21, becomes autonomous, and was wrongly taken for a 'dark' galaxy.

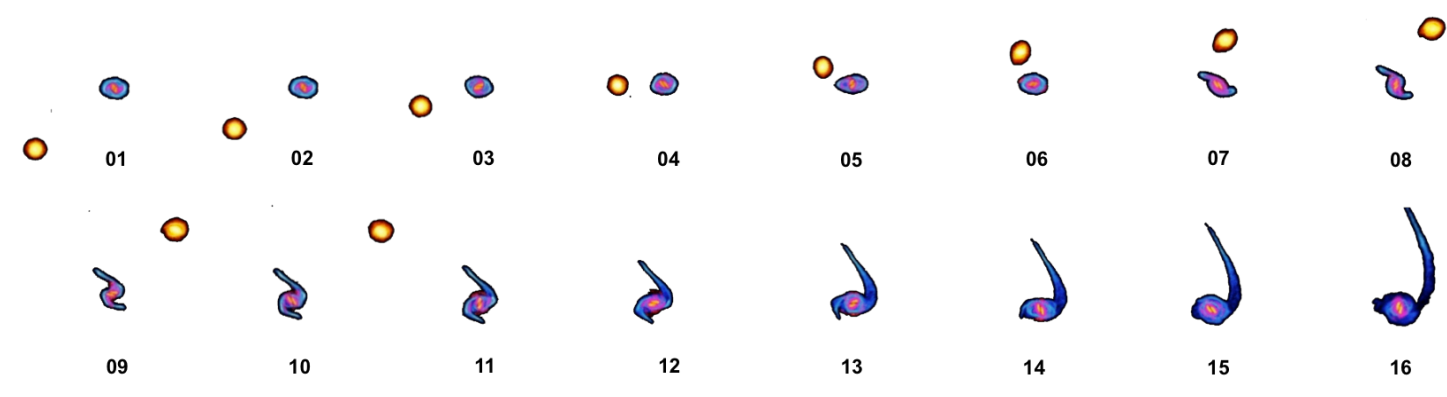

Figure 2. Simulation of VirgoHI21 formation.

The case of VirgoHI21 illustrates a third feature that simulation and experiment share. Both sometimes make it possible to visualize a representation of the system under study. In the example, we can see the time evolution of the reproduced collision. While the simulation of VirgoHI21 formation is derived from physical laws, it also becomes concrete, on screen, for the simulationists.

\subsection{Time Evolution}

The case of VirgoHI21 also illustrates a fourth feature that simulation and experiment may share. They both sometimes show the time evolution of the system being studied. Simulations are even better than experiments in doing this since, unlike experiments, 
they allow the scientists to see the time evolution of the system under study at whatever the time scale that characterizes it. For instance, a natural phenomenon can be too slow to be observed, e.g. the collision of two galaxies. In this case, the simulation can help one to visualize the phenomenon by speeding up its natural temporality. Of course, this can also be done by recording an experiment, and watching the film by speeding up it, but some phenomena such as the collision of two galaxies last too long to be entirely filmed.

\subsection{Black Box Effect}

There is a question whether simulation and experiment both function as black boxes, and therefore whether this is another similarity between them. Guala (2002) suggests that only experiments may act as black boxes, whereas Dowling and Suchman, an anthropologist and sociologist, argue that they both sometimes do. I will present the two positions.

An experiment functions as a black box when the experimenter does not know some (or all) of the physical processes at work in the observed phenomenon. By contrast, in the case of simulations, the simulationist can in principle know the content of the computer program. Nothing in the program is prima facie hidden from her. Is it, as Guala (2002) thinks, a reason why simulations are not black boxes?

According to Guala, in order to conduct an experiment, it is not required to possess a complete knowledge of the physical processes at work in the target system.

On his view, ignorance is the very reason why the experiment is done. He writes, 'Experiments are particularly useful when one has an imperfect understanding of some basic causal mechanism of the system under study. They can be used in these contexts because the laboratory "stuff" is assumed to be the same as the non-laboratory "stuff", (Guala 2002, 11).

Thus, for Guala, an experimental system must share the same physical processes (or 'the same causal mechanisms') with the target system. To this end the experimental system must be made of the same matter as the target system; it must have the same relevant material features of the target system. Only in this way can the conclusions from the experiment can be extended to the target system. As Guala asserts, 'Of course, quite a lot of knowledge is required in order to do so, but no fundamental theory of how 
the target system works is needed. Parts of the laboratory system can be put between brackets and used as "black boxes"” (Guala 2002, 12).

In other words, we do not need to know in advance the laws that describe the behaviour of the experimental system and the target system. For Guala, this is a fundamental difference between simulations and experiments as 'the knowledge needed to run a good simulation is not quite the same as the one needed to run a good experiment' (Guala 2002, 12).

According to Dowling and Suchman, simulations also often function as black boxes. The main reason here is that simulated phenomena are still the results of long and complex calculations that cannot be mentally surveyed by a human mind. In the case of simulations, this is due to the complexity of the computer program and the speed of the computational process on the machine. Dowling writes, 'The results of a simulation are unpredictable. They are typically based on calculations that are analytically intractable, and the computer's numerical computation of the solutions is far too rapid to be followed by an individual scientist. The complexity and unpredictability of a computer program invite the scientist to present it as a black box, interacting with it in an experimental manner, trying things out to see what will happen' (Dowling 1999, 266). Thus, even though the simulationist knows the content of the computer program, she will hardly be able to anticipate the results or grasp how they have been obtained from it.

I believe that Dowling's and Suchman's stance does not straightforwardly contradict Guala's. They found another origin of treating simulations as black boxes. This is therefore for distinct reasons that a scientist may adopt the same epistemic attitude of ignorance facing a simulation or an experimental system as if they were black boxes. On one side, for Guala, the reasons are that scientists are ignorant concerning some (or all) of the physical processes at work in an experiment. On the other, for Dowling and Suchman, the reasons are that the scientists cannot mentally grasp the calculations.

\subsection{Similarities in Knowledge Generation}

Now that I have reviewed similarities between simulations and experiments that are often highlighted in the literature-i.e. that they allow for exploration, intervention, and 
visualization, that they sometimes unfold over time, and that they function as black boxes-I will discuss whether these features allow simulations to generate new knowledge.

First, simulations produce knowledge about the target system if the simulation model is an accurate representation of the target system. It is because simulation models contain the appropriate equations that they can provide knowledge. And yet none of the five similarities plays a role in the representational function of the simulation model. One can explore the consequences of a simulation model and intervene on a computer program even if the model does not represent anything empirical. This is the case for simulations that do not instantiate empirical systems, like the simulation of Game of Life. The Game of Life created by mathematician John H. Conway in 1970 is not supposed to represent an empirical system. It is a two-dimensional grid of square cells. Each cell is either in the state 'live' or in the state 'dead'. A cell's state at time $t+1$ depends on its state at time $t$ and the states of its eight neighbours following three simple rules described in Berlekamp, Conway, and Guy (2004, 927):

i) A cell that's dead at time t becomes live at $t+1$ only if exactly three of its eight neighbours were live at $t$;

ii) A cell that's live at $t$ and has four or more of its eight neighbours live at $t$ will be dead by time $t+1$;

iii) A live cell that has only one live neighbour, or none at all, at time $t$, will also be dead at $t+1$.

The same is true with the three other similarities: that simulations provide lifelike images, present a temporal dimension or function as black boxes does not ensure that the model correctly represents the target system and therefore these features do not make simulations produce knowledge. For instance, video games make it possible to visualize objects over time and are also black boxes in that the player does not have access to the program behind the games. Nevertheless video games do not provide any knowledge about the animated objects.

Furthermore the five features do not bestow any knowledge-making capability upon experiments. In experiments, knowledge is obtained from empirical data if these data are generated by an experimental system that contains the same relevant properties 
as the target system, given the particular question the scientist wants to answer about the target system. If the experimental system does not possess the relevant material characteristics of the target system, which can explain the phenomenon under study, one cannot draw the right conclusions from the experiment. In order to illustrate this point, let us assume that biologists have to test a new drug on rats in the hope of curing humans from some disease. If the rats' reaction to the treatment depends upon a physiological pathway that is absent in humans, we would wrongly extend the experiment's results to our species. (This example is given by Parker 2009, though for a different purpose.)

As shown, it is not in virtue of the similarities commonly highlighted in the philosophical literature that simulations and experiments can be said to provide new knowledge. Thus there is no analogy that can account for the novelty claim. That said, it certainly does not follow that computer simulation cannot produce new knowledge. What is the widely shared intuition behind the novelty claim then? In the next section, I will suggest that simulations are often associated with experiments by scientists because the features that simulations share with experiments invite the simulationist to interact with simulations in an experimental manner.

\section{Experimental-like Interactions with Simulations}

While the five similarities between simulations and experiments do not give simulations any knowledge-making capability, they still impact the way scientists interact with simulations and experiments. They make interactions with simulations experimentallike. Simulationists thus learn how to draw inferences from computer simulations in a way that may be similar as the way experimenters learn how to draw inferences from experiments. Consequently simulationists may develop skills similar to those of experimenters. This is what I want to show in this section.

As I have said, simulations, like experiments, allow for both exploration and intervention. Thus the simulationist (or the experimenter) learns how to control and to modify the behaviour of the simulated (or experimental) system in adequately modifying the input parameters of the computer program (or the experimental setup). Both the simulationist and the experimenter also get used to recognizing regular 
behaviours of the system and to anticipating the response of the system to a modification of a parameter. For example, if the target system is a plane flying against wind force, the simulationist may learn how to anticipate the plane speed variations against different force values, by visualizing on screen the modelled plane, and the experimenter by observing a concrete model of the plane in a wind tunnel. As a result, the simulationist may develop skills similar to those of an experimenter.

As Dowling claims, based on interviews with simulationists, 'A sense of direct manipulation encourages simulators to develop a "feel" for their mathematical models with their hands and their eyes, by tinkering with them, noticing how they behave, and developing a practical intuition for how they work' (Dowling 1999, 269). So it comes as no surprise that the scientists might interact with the simulative system in the same way they would have interacted with the actual target system itself. Dowling even claims that 'the simulation is presented as an experimental target', and, because of this, 'the researcher can interact with it as if it were a "real" target, drawing on the physical skills of recognition and reaction' (Dowling 1999, 269).

Visualization is another experimental-like aspect in that the simulationists are inclined to manipulate the simulative system the same way they would manipulate the actual target system. Visualization makes simulated phenomena concrete for the simulationists and therefore allows a sense of direct manipulation. As Turkle, a sociologist and psychologist, and Papert, a mathematician and computer scientist, write, 'The computer stands betwixt and between the world of formal systems and physical things; it has the ability to make the abstract concrete. In the simplest case, an object moving on a computer screen, might be defined by the most formal of rules and so be like a construct in pure mathematics; but at the same time it is visible, almost tangible, and allows a sense of direct manipulation' (Turkle and Papert 1991, 162). Of course, it would be excessive to claim that the simulationists forget that simulations are not 'real' phenomena. The fact that the simulated phenomena are generally displayed on screen makes the confusion impossible, particularly when they are displayed under more vivid and artificial colours and under more simple geometrical forms than the actual phenomena in nature.

Furthermore, because the simulation becomes concrete for the simulationist, it appeals to her hard-wired perceptual capacities and skills, the same ones that an experimenter uses in the process of discovery. This seems particularly true when the 
simulation pictures provide lifelike images that look like the corresponding photographs. For example, in Figure 1, the simulation picture looks like the actual photograph of VirgoHI21 in that they share many visual attributes, e.g. the same colours, and the same patterns at the same locations. Winsberg also endorses this idea; he writes that 'psychologically, at the very least, working with a simulation is much more like doing an experiment if the simulation produces lifelike images reminiscent of laboratory photographs' (Winsberg 2003, 110).

The fact that simulation unfolds over time is another aspect that may also make the simulationist experience the simulation the same way she would experience an experiment. She may see the time evolution of the simulated phenomenon the same way she would have observed the evolution of the target system. The temporal dimension of simulation appeals to her cognitive capacities of treating and gaining knowledge on time from visualized phenomena that are similar to the ones that an experimenter uses.

Lastly, that a simulation functions as a black box also contributes to make interactions with simulations experimental-like. Because sometimes unpredictable results seem to come out of the computer program, the simulationist may see it as a black box and, for Dowling, this allows the scientist to ignore the internal structure of the simulations program and to interact with this program the way she would have interacted with the experimental system. 'The unpredictability of a simulation run makes the activity of simulation similar to the activity of performing a physical experiment. The scientist prepares the system, sets initial conditions, then takes a relatively passive role, waiting to find out how the system will respond' (Dowling 1999, 265). Suchman also writes, 'Insofar as the machine is somewhat predictable, in sum, and yet is also both internally opaque and liable to unanticipated behaviour, we are more likely to view ourselves as engaged in interaction with it than as just performing operations upon it, or using it as a tool to perform operations upon the world' (Suchman 1987, 16). Through her interactions with the computer program, the scientist focuses her attention on the simulated phenomenon itself, and thus learns 'how things work'. In other words, the 'black box' effect invites the simulationist to experience the simulation the way she would experience an experiment. Like an experimenter in front of an experimental device, the simulationist waits for what will result from the simulation. She changes the simulation parameters and observes on screen how the simulative system reacts to her modifications. That said, as Dowling points out, the black box 
effect is only temporary. When the simulationist deals with the reliability of the simulation, she does not see it as an experiment anymore but rather as a product of the simulation model. Based on interviews with scientists, Dowling notices that they consider a simulation as the product of the model when they discuss the design, the accuracy or the solubility of the equations; while they consider it as an experiment when they discuss what they visualize on screen.

In a nutshell, the features that simulations share with experiments have nothing to do with new knowledge generation itself, but affect the way scientists draw inferences from them. This can explain why scientists often use the expression 'numerical experiment' to designate a computer simulation (or 'experimental simulation', 'simulated experiment', 'in silico experiment'). Therefore we should acknowledge that, from the point of view of scientists at least, simulations are more than mere calculations, and that they share some noticeable features with experiments, which make them experimental-like in that way.

\section{Novelty in Simulations and Experiments}

The similarities between simulations and experiments do not allow to ground any analogy that can account for the novelty claim, although they may explain the intuition behind. In this last section, I do not discuss any further possible features that would bestow any knowledge-making capability upon simulations and experiments. I will assume that simulation models and experimental systems are appropriate representations of the target systems for reasons I will not elaborate. I now want to suggest that computer simulations and experiments yield most of the time new knowledge under the same epistemic circumstances, independently of the features they may share. For that, I need to define a criterion for novelty. I summarize the arguments in this section in Table 1.

First I shall make it clear that I will focus on scientific domains where theories are available. Barberousse and Vorms make an important distinction between two cases: (i) the 'terra incognita' context 'when the information about the domain of phenomena is scarce, and when systematic exploration is the only way to obtain it' (Barberousse and Vorms 2013, 40), and (ii) the availability of a theoretical framework which is 'the 
set of all available knowledge about the domain of phenomena at hand, be it of theoretical or empirical origin' (Barberousse and Vorms 2013, 41). In the terra incognita context, only experiments can provide new knowledge; by default, novelty is here surprising since scientists have no expectations about what they are going to learn. Here simulations cannot provide new knowledge simply because there is a lack of theories. The reason is therefore not because simulations are unable to yield new knowledge. That is why I shall focus on the second case: when a theoretical framework is available, computer simulations may provide new knowledge, even though, Barberousse and Vorms claim, they are less likely to yield 'surprising' novelty (an exception they provide is the computer-aided study of deterministic chaos).

I now consider the 'non-entailment view' as an objection against the novelty claim. I will argue that the criterion for novelty underlying the non-entailment view is irrelevant since it does not even account for what scientists consider in practice as being new in experiments. I will then offer a criterion for novelty.

According to the non-entailment view (Lusk 2016, 151), simulations do not produce any new knowledge because they are calculations, and simulation outputs are logical consequences of the theoretical assumptions contained in the simulation model. Non-entailment is notably supported by Beisbart and Norton when they say Monte Carlo simulations 'can only return knowledge of the world external to them in so far as that knowledge is introduced in the presumptions used to set up the simulation' (Beisbart and Norton 2012, 404). It is here suggested that (i) in an experiment, 'nature can "push back" with results that are both conceptually and ontologically distinct from the experimental setup' (Morrison 2015, 249); and (ii) a computer simulation 'only gives us the consequences of what has been programmed into the computer' (Morrison 2015, 249). In other words, only experiments, because they involve physical interactions, would be likely to yield new empirical data. Simulation outputs are virtually contained in the model assumptions, and simulations only make the consequences of these assumptions explicit. Of course revealing what is only implicitly contained in the simulation model is a valuable achievement and may produce surprising results, but it brings no new knowledge.

In the non-entailment view, the following criterion for novelty is thus suggested: a piece of knowledge is new if it is not already (explicitly or implicitly) contained in the available theory. 
According to the non-entailment criterion, when experiments yield results that a well-confirmed theory already predicts, they do not offer new knowledge. Theories generally precede empirical investigation. It is often the case that, before running an experiment, the experimenter performs theoretical calculations and therefore has an expectation about the experimental results she is going to measure or the observations she is going to make. For example, the discovery of the Higgs boson is precisely what the standard model predicts, and therefore is redundant and not new following the nonentailment criterion - although it brings a much valuable piece of information, namely that the standard model is correct.

In accordance with this criterion, experiments produce new knowledge only if their results contradict the theoretical assumptions that allow for their production. The contradiction may lead to the revision of the theoretical assumptions. For example, the observations of planets' positions and axial precession contradicted Ptolemaic astronomy and confirmed Newton's theory; the observation that the weight of some metals increases when they are intensively warmed contradicted the phlogiston theory and argued in favour of Lavoisier's theory. However theoretical conflicts are not business as usual in science, which makes the non-entailment criterion irrelevant. My argument is framed in terms of frequency: conflicts between theory and experiment, which according to Kuhn lead to revolutions, do not happen frequently in the history of science in comparison with the intensive use of simulations and experiments and the number of successes of theories in predicting phenomena. Scientists use simulations and experiments all the time to gain knowledge about the empirical word. And, in terms of frequency, scientists are more likely to get confirmation than refutation.

To put it in another way, it is not usual for an experiment to confound a scientist, although only an experiment can do that while a simulation cannot. There is confusion (Morgan 2003) when a result is provided which was not expected, and that the available theory does not explain at all. The discovery of X-rays illustrates perfectly this case. This discovery was a genuine coincidence that certainly confounded the physicist Röntgen 'on the day that [he] interrupted a normal investigation of cathode rays because he had noticed that a barium platoon-cyanide screen at some distance from his shielded apparatus glowed when the discharge was in process' (Kuhn [1962] 1996, 57). This kind of empirical discovery, which contradicts the available theory and therefore leads to confusion, is less frequent than the successes of a theory in predicting phenomena. 
The non-entailment criterion relies on what one can draw in principle from available theories, and falls short in even accounting for novelty in experiments. In practice, knowledge is considered as new by scientists when it enlarges the set of existing knowledge. Therefore I suggest the following criterion for novelty-I shall call it the 'first time' criterion: a simulation or an experiment provides new knowledge when this knowledge is obtained for the first time and is added in a relevant way to existing knowledge. Here novelty is clearly context dependent.

I want now to argue that simulations and experiments generally provide new knowledge under the same epistemic circumstances, i.e., when the 'first time' criterion is met. Simulation outputs are considered to be new if they have been obtained under physical conditions that have not yet been explored. For example, the existence of a material harder than diamond was a computer-aided discovery (Liu and Cohen 1989). As it was first discovered by a simulation, this simulation provided genuinely new knowledge in this criterion. This discovery preceded the display of empirical evidence, namely the successful production of two materials harder than diamond: wurtzite boron nitride and lonsdaleite (Pan et al. 2009). In this case, computer simulations have led to a new finding, as the empirical data-i.e. the existence proof and the measurement of indentation strength of wurtzite boron nitride and lonsdaleite-were unknown to scientists.

A simulation result that is expected or is similar to another previous result cannot be considered to be new according to the 'first time' criterion, however. In a similar way, an experiment does not offer any novelty if it has been reproduced and its results were already known (e.g., because they are indicated in a scientific handbook) or if the highly confirmed theory has already been used to predict these same results. In this sense, we generally would not say that experimental data are new if the conditions under which they have been obtained are similar to conditions that have already been explored.

On the other hand, the 'first time' criterion for novelty is relevant since it corresponds to usual practices: a simulationist runs a simulation because she wants to gain knowledge about the target system; precisely because this knowledge is not known beforehand. Thus she expects to be surprised (although not confounded; Morgan 2003). It would be tempting, Morgan (2003, 220-221) claims, to believe that only experiments are likely to surprise us because, in the case of simulations, the answers to the questions 
that we raise are 'already designed in the model', and are thereafter revealed by calculation. On the contrary, as Morgan emphasizes, an experiment or a simulation can surprise the scientist in the sense that they offer a result which was not expected, but that the available model can explain (for a recent analysis of the concept of surprise in this context, see Parke 2014). For instance, in the example of VirgoHI21, the observation of this false dark galaxy surprised the astrophysicists because it seemed at first glance to contradict the current theory, while it has subsequently been theoretically explained by simulation. Surprise, I shall emphasize, is also produced by the black box effect. A simulation result can be deemed surprising insofar as the scientist cannot predict it by herself. She would not be able to deduce this result alone without the machine.

Of course the fact that, most of the time, simulations and experiments provide new knowledge in a similar way (i.e., in accordance with the 'first time' criterion) should not lead us to deny the special place of experiments in science. Making refutation possible alone legitimates the unique role of experiments. An experiment, when it is well run, can contradict our best available theory, whereas a simulation cannot constitute a refutation of the theory. The simulation can increase our trust in a theory as a good prediction does, as it is the case in the example of VirgoHI21. If the scenario of VirgoHI21 formation had not made it possible to find a final simulation picture similar to the telescope photograph, the astrophysicists would certainly study other scenarios. If in turn these new scenarios failed, the astrophysicists would have questioned the model's simplifying assumptions, or worse, the theoretical assumptions themselves. In the last case, the simulation would have helped to test scenarios, but the observation alone would have contradicted the theory.

\begin{tabular}{|c|c|c|c|c|c|}
\hline & \multicolumn{2}{|c|}{ "Terra incognita" context } & \multicolumn{3}{|c|}{ Availability of a theoretical framework } \\
\hline & & & \multicolumn{2}{|c|}{ Non-entailment view } & First time criterion \\
\hline & unsurprising & surprising & unsurprising & surprising & surprising by default \\
\hline experiments & $\varnothing$ & $\checkmark$ & $\begin{array}{c}\varnothing \\
(a)\end{array}$ & $\begin{array}{l}\checkmark \\
\text { (b) }\end{array}$ & $\checkmark$ \\
\hline $\begin{array}{l}\text { computers } \\
\text { simulations }\end{array}$ & $\varnothing$ & $\varnothing$ & $\varnothing$ & $\begin{array}{l}\varnothing \\
\text { (c) }\end{array}$ & $\checkmark$ \\
\hline
\end{tabular}

Table 1. (a) Agreement with theory; (b) (rare) contradictions with theory, creating 'confusion'; (c) exception: deterministic chaos. 


\section{Conclusion}

I have first reviewed some similarities between simulations and experiments commonly highlighted in the philosophical literature: they both allow for exploration, intervention and visualization, they sometimes unfold over time and they may function as black boxes. I then have shown that these features are not what allow simulations and experiments to generate new knowledge.

Instead, I have argued that they shape the way scientists draw inferences, which is experimental-like. I have suggested that this may explain the widely shared intuition behind the novelty claim.

I have then analysed the concept of novelty and, from this, have framed an argument that simulations and experiments generally provide new knowledge under the same epistemic circumstances, independently of the features they may share; this is a way of making sense of the novelty claim. They provide new knowledge when they are obtained for the first time and enlarge in a relevant manner the set of existing knowledge. Only experiments can provide new knowledge in the sense that they contradict the theory. Even though this possibility legitimates the special place of experiments in science, this is nevertheless not business as usual in science.

\section{Acknowledgements}

I am very grateful to Anouk Barberousse, Thomas Boyer-Kassem, Paul Humphreys, Cyrille Imbert, Ashley Graham Kennedy, and John Stewart, as well as to the two anonymous referees of this journal for their helpful comments. Any remaining shortcomings are of course mine. 


\section{References}

Barberousse, A., S. Franceschelli, and C. Imbert. 2009. "Computer Simulations as Experiments." Synthese 169: 557-574.

Barberousse, A., and M. Vorms. 2013. “Computer Simulations and Empirical Data." In Computer Simulations and the Changing Face of Scientific Experimentation, edited by J. M. Durán and E. Arnold, 29-45. Newcastle upon Tyne: Cambridge Scholars Publishing.

Beisbart, C., and J. D. Norton. 2012. "Why Monte Carlo Simulations Are Inferences and Not Experiments." International Studies in the Philosophy of Science 26: 403-422.

Berlekamp, E. R., J. H. Conway, and R. K. Guy. 2004. Winning Ways For Your Mathematical Plays. 2nd ed. Wellesley, MA: A. K. Peters.

Dowling, D. 1999. "Experimenting on Theories." Science in Context 12: 261-273.

Duc, P.-A., F. Bournaud, and E. Brinks. 2007. "Tidal Debris Posing as Dark Galaxies.” In Dark Galaxies and Lost Baryons, edited by J. I. Davies and M. J. Disney, 216225. Paris: International Astronomical Union.

Galison, P. 1996. “Computer Simulations and the Trading Zone.” In The Disunity of Science: Boundaries, Contexts, and Power, edited by P. Galison and D. J. Stump, 118-157. Stanford, CA: Stanford University Press.

Giere, R. N. 2009. "Is Computer Simulation Changing the Face of Experimentation?" Philosophical Studies 143: 59-62.

Guala, F. 2002. "Models, Simulations, and Experiments." In Model-based Reasoning: Science, Technology, Values, edited by L. Magnani and N. J. Nersessian, 59-74. New York: Kluwer.

Guala, F. 2005. The Methodology of Experimental Economics. Cambridge: Cambridge University Press.

Hacking, I. 1983. Representing and Intervening: Introductory Topics in the Philosophy of Natural Science. Cambridge: Cambridge University Press.

Humphreys, P. 1994. “Numerical Experimentation.” In Patrick Suppes: Scientific Philosopher, edited by P. Humphreys, vol. 2, 103-121. Dordrecht: Kluwer. Humphreys, P. 2004. Extending Ourselves: Computational Science, Empiricism, and Scientific Method. New York: Oxford University Press. 
Jebeile, J. 2016. “Les simulations sont-elles des expériences numériques?” Dialogue 55: $59-86$.

Keller, E. F. 2003. "Models, Simulation, and 'Computer Experiments'.” In The Philosophy of Scientific Experimentation, edited by H. Radder, 198-215. Pittsburgh, PA: University of Pittsburgh Press.

Kuhn, T. S. [1962] 1996. The Structure of Scientific Revolutions. 3rd ed. Chicago, IL: University of Chicago Press.

Liu, A. Y., and M. L. Cohen. 1989. "Prediction of New Low Compressibility Solids." Science 245: 841-842.

Lusk, G. 2016. "Computer Simulation and the Features of Novel Empirical Data." Studies in History and Philosophy of Science 56: 145-152.

Morgan, M. S. 2002. "Model Experiments and Models in Experiments." In Modelbased Reasoning: Science, Technology, Values, edited by L. Magnani and N. J. Nersessian, 41-58. New York: Kluwer.

Morgan, M. S. 2003. "Experiments without Material Intervention: Model Experiments, Virtual Experiments, and Virtually Experiments." In The Philosophy of Scientific Experimentation, edited by H. Radder, 216-235. Pittsburgh, PA: University of Pittsburgh Press.

Morgan, M. S. 2005. “Experiments versus Models: New Phenomena, Inference, and Surprise." Journal of Economic Methodology 12: 317-329.

Morrison, M. 2009. "Models, Measurement and Computer Simulation: The Changing Face of Experimentation." Philosophical Studies 143: 33-47.

Morrison, M. 2015. Reconstructing Reality: Models, Mathematics, and Simulations. Oxford: Oxford University Press.

Norton, S. D., and F. Suppe. 2001. "Why Atmospheric Modeling Is Good Science.” In Changing the Atmosphere: Expert Knowledge and Environmental Governance, edited by C. A. Miller and P. N. Edwards, 67-106. Cambridge, MA: MIT Press.

Pan, Z., H. Sun, Y. Zhang, and C. Chen. 2009. "Harder than Diamond: Superior Indentation Strength of Wurtzite BN and Lonsdaleite." Physical Review Letters 102: 055503.

Parke, E. C. 2014. "Experiments, Simulations, and Epistemic Privilege." Philosophy of Science 81: 516-536. 
Parker, W. S. 2009. “Does Matter Really Matter? Computer Simulations, Experiments, and Materiality." Synthese 169: 483-496.

Radder, H. 1996. In and About the World: Philosophical Studies of Science and Technology. Albany: State University of New York Press.

Rohrlich, F. 1991. "Computer Simulation in the Physical Sciences." In PSA 1990: Proceedings of the 1990 Biennial Meeting of the Philosophy of Science Association, edited by A. Fine, M. Forbes, and L. Wessels, vol. 2, 507-518. East Lansing, MI: Philosophy of Science Association.

Sismondo, S. 1999. "Models, Simulations and Their Objects." Science in Context 12: $247-260$.

Suchman, L. A. 1987. Plans and Situated Actions: The Problem of Human-Machine Communication. Cambridge: Cambridge University Press.

Tiles, J. E. 1993. “Experiment as Intervention.” British Journal for the Philosophy of Science 44: 463-475.

Turkle, S., and S. Papert. 1991. "Epistemological Pluralism and the Revaluation of the Concrete." In Constructionism: Research Reports and Essays 1985-1990, edited by I. Harel and S. Papert, 161-191. Norwood, NJ: Ablex.

Winsberg, E. 2003. "Simulated Experiments: Methodology for a Virtual World." Philosophy of Science 70: 105-125.

Winsberg, E. 2009. “A Tale of Two Methods.” Synthese 169: 575-592.

Woodward, J. 2003. Making Things Happen: A Theory of Causal Explanation. New York: Oxford University Press. 